\title{
Evolution de la réglementation des eaux minérales naturelles
}

\author{
The evolution of the mineral water regulation \\ par E. Papciak \\ Division Nationale des Eaux Minérales et Thermales \\ Secrétariat d'État à l'Industrie
}

We known that mineral waters have been being used in France since the Gallo-Roman period. Some ordinances and patent letters of the 15th century and later show that the government wanted to control the spring exploitation. But the foundations of the regulation were defined in the 19th century when the bottling industry developed. The legislation firstly gives attention to the resource protection and to the spring exploitation conditions, Later, it adapts to sanitary constraints. Nowadays, it must integrate those of the European and international legislation: European directives, Codex Alimentarius standards,...

\section{I - LES EAUX MINÉRALES SOUS L'ANCIEN RÉGIME}

L'utilisation des eaux chaudes d'origine souterraine à des fins thérapeutiques était déjà connue depuis l'antiquité chez les Égyptiens, Grecs, Romains et Celtes.

En France, après une longue période sombre qui a suivi l'invasion des barbares, de nombreux écrits de la Renaissance font état de la fréquentation des bains par des personnalités distinguées, notamment dans les Pyrénées (Cauterets, Eaux-Bonnes, Bagnères), dans le Bourbonnais (Néris, Bourbon l'Archambault, Saint Pardoux, Vichy, Bourbon Lancy) et dans les Vosges (Plombières).

Les premiers textes officiels à caractère réglementaire, semblent être les lettres patentes de 1549 (Henri II) selon lesquelles les sources thermales, comme toutes autres sources, appartiennent à l'État. Mais cette disposition, bien que confirmée à plusieurs reprises, notamment en 1687 et 1694 par Louis XIV, n'est pas respectée avec rigueur si bien que de nombreux propriétaires privés se permettent d'exploiter des eaux minérales avec la tolérance bienveillante des contrôleurs royaux. Cette tolérance est même reconnue officiellement dans une déclaration du 25 avril 1772. Après avoir rappelé que "c'est toujours le Roi qui se réserve l'exploitation des sources", cette déclaration "respecte le droit des propriétaires qui sont en possession de sources et fontaines sur leurs terrains, à charge, pour eux, de se soumettre aux mesures de police prescrites". Ce problème de droit sur les sources est définitivement réglé avec l'arrêt du Conseil d'État du 5 mai 1781 qui, pour la première fois, fait la distinction entre les sources appartenant à l'État et celles découvertes par des particuliers sur leurs terrains, ces derniers ayant la possibilité de les exploiter sous réserve de se soumettre aux règlements et de disposer préalablement d'une autorisation délivrée par la Société de Médecine.

De nombreux autres textes sont publiés au coup par coup, sur le fonctionnement des établissements de soins, sur le rôle des inspecteurs et sur les redevances que les établissements doivent verser. Tous ces textes ont beaucoup évolué et se sont structurés aux $\mathrm{XIX}^{\mathrm{e}}$ et $\mathrm{XX}^{\mathrm{e}}$ siècles. Nous allons les examiner sous deux aspects :

- conditions d'exploitation de l'eau minérale naturelle,

- protection de la ressource.

\section{II - RÉGLEMENTATION APPLICABLE À L'EXPLOITATION DES SOURCES D'EAUX MINÉRALES NATURELLES}

Le texte de base de la réglementation, en vigueur encore actuellement, est une ordonnance du 18 juin 1823 qui synthétise l'ensemble des règlements parus antérieurement. Elle rappelle en premier lieu que "toute entreprise ayant pour effet de livrer ou d'administrer au public des eaux minérales naturelles ou artificielles, demeure soumise à une autorisation préalable et à l'inspection d'hommes de l'art". Cette ordonnance a été ensuite complétée par de nombreux textes réglementaires qui ont précisé les critères auxquels doit satisfaire une eau minérale, les conditions de contrôle et d'inspection et les procédures préalables à la délivrance d'autorisation. 


\subsection{Critères applicables aux eaux minérales naturelles}

Pendant longtemps la définition de l'eau minérale est restée imprécise. Les auteurs du "Dictionnaire des eaux minérales et d'hydrologie médicale" de 1860 avaient proposé de retenir la définition suivante : "On doit entendre par eaux minérales toutes celles qui, en raison soit de leur température bien supérieure à celle de l'air ambiant, soit de la quantité et de la nature de leurs principes salins et gazeux, sont ou peuvent être employées comme agent médicamenteux". Le caractère thérapeutique apparaît comme élément fondamental. D'ailleurs, il a été affirmé pour la première fois dans la dénomination légale de l'arrêt du Conseil d'État du 5 mai 1781 dans lequel les termes "eaux minérales et médicinales" sont répétés à plusieurs reprises de manière indissociable.

Dans cette définition, qui a été reprise dans une circulaire de 1922, l'origine souterraine de l'eau n'apparaît pas comme critère obligatoire, pour la simple raison que les "eaux minérales artificielles" font partie de la catégorie des "eaux minérales". L'origine souterraine est exigée uniquement pour les "eaux minérales naturelles" qui sont considérées comme un don de la nature et qui acquièrent leurs caractéristiques (minéralisation, température) naturellement au cours de leur parcours souterrain.

Le caractère thérapeutique était attaché à la pureté originelle de l'eau. Son utilisation comme agent médicamenteux ne tolérait pas la moindre transformation : elle ne pouvait être livrée au public que telle qu'elle se présentait à l'émergence. Ceci a été affirmé dans une circulaire ministérielle du 4 décembre 1894 qui a prescrit que chaque demande en autorisation soit "accompagnée d'un certificat du service des mines attestant que l'eau n'est soumise à aucune opération de décantage ou de gazéification", le pétitionnaire devant prendre "l'engagement de ne faire subir à l'eau aucune de ces manipulations". Cette exigence a été ensuite abandonnée. Après une période de tolérance, le décret du 28 mars 1957 a admis qu'il était possible d'autoriser par arrêté la "séparation d'éléments instables, par décantation ou filtration, éventuellement précédée d'une oxygénation", la dégazéification, l'incorporation ou la réincorporation de gaz carbonique, sous réserve que ces traitements n'aient pas pour but ou effet de modifier les caractéristiques microbiologiques de l'eau.

\subsection{Contrôle et inspection des sources et des établissements}

L'ordonnance de 1823 confiait l'inspection des établissements de distribution d'eau minérale uniquement à des "docteurs en médecine ou en chirurgie" nommés par le ministre. Mais, quelques années plus tard, est apparue la nécessité de faire appel également aux ingénieurs ayant une bonne connaissance dans le domaine du sous-sol. Le ministre recommande donc aux préfets de faire appel, en cas de besoin, aux ingénieurs des mines. Le rôle de ces derniers est d'ailleurs officialisé par la loi du 14 juillet 1856 et par les décrets des 8 septembre 1856, 28 janvier 1860 et 14 août 1869, ce dernier traitant spécialement des attributions du "service des mines".

Les "docteurs en médecine" ont été remplacés en 1860 par des "médecins inspecteurs" puis par des inspecteurs départementaux d'hygiène en 1930.

A la fin du XIXe siècle, les autorités commencent à s'intéresser à la qualité bactériologique de l'eau distribuée dans les établissements. Une circulaire à ce sujet est envoyée aux préfets le 26 janvier 1891. Les risques de pollution des sources par des agents extérieurs sont évoqués dans les circulaires de 1894 et 1895 , mais les analyses physico-chimiques et microbiologiques sont prescrites seulement par décret du 6 août 1908. Il ne s'agit pas encore de contrôles systématiques. Ils le deviennent seulement à partir de 1930 avec la parution du décret du 30 avril qui impose pour chaque source au moins deux analyses bactériologiques par an, pratiquées par un laboratoire agréé. Les modalités d'application de cette disposition ainsi que la liste des laboratoires agréés sont précisées par l'arrêté du 14 octobre 1937. D'autres contrôles analytiques périodiques sont venus ensuite s'ajouter au niveau des points d'usage dans les établissements thermaux et sur les chaînes d'embouteillage.

\subsection{Autorisations administratives préalables à l'exploitation d'une source d'eau minérale}

L'autorisation prévue par l'ordonnance de 1823 prend la forme d'un arrêté ministériel, la formule généralement employée étant très laconique : "est autorisée l'exploitation de la source d'eau minérale naturelle ... alimentant l'établissement situé sur la commune de ....". La ou les sources sont parfois désignées par leur nom, mais ce n'est pas toujours le cas.

Les conditions de délivrance de l'autorisation ne sont pas très précises. A l'article 2 de l'ordonnance, il est seulement indiqué que les autorisations sont délivrées "sur l'avis des autorités locales, accompagné, pour les eaux minérales naturelles, de leur analyse...". La délivrance d'autorisations n'est soumise à une procédure un peu plus élaborée qu'à partir de 1930 (3ème décret du 30 - 4-1930).

Les autorisations étant accordées, les propriétaires sont tenus de veiller, sous le contrôle des inspecteurs, à leur conservation et à leur amélioration. A partir de 1855 , ce rôle est confié aux ingénieurs des Mines qui sont chargés également de diriger l'exécution des projets d'amélioration. Il faut noter d'ailleurs que ces projets d'amélioration peuvent prendre des formes très variées : curage et restauration des puits, recaptage par des ouvrages distincts (puits ou forage) situés à proximité... La circulaire du 15 octobre 1855 stipule que "lorsque sur les sources appartenant à l'État, aux départements, aux communes ou établissements publics, des travaux de captage, de recherche ou d'aménagement s'exécuteront, MM. les ingénieurs devront surveiller cette exécution et rendre immédiatement compte à l'administration locale ou à l'administration supérieure, suivant les cas, des faits qui paraîtront devoir attirer son attention." Pour les sources appartenant à des propriétaires privés, ce compte rendu de travaux ne semble pas être exigé.

Cette situation perdure pratiquement jusqu'en 1957. Les règlements n'imposent même pas, après ces travaux d'amélioration, de réviser l'autorisation initiale, sauf pour le cas de modification de la minéralisation de l'eau. C'est ce qui ressort de la circulaire du 30 mai 1914 qui, après avoir rappelé que "les eaux minérales présentant le caractère d'un véritable médicament, il importe que leur composition reste constante" et qu'en cas de modification de la minéralisation constatée par le propriétaire, ce dernier est tenu de faire parvenir un échantillon à l'Académie de Médecine qui, après l'avoir analysé, décide "si l'inscription d'une nouvelle formule dans son Bulletin constitue une mesure suffisante, ou, si en présence d'une modification profonde de la composition de l'eau, il convient de procéder à une nouvelle enquête technique." 


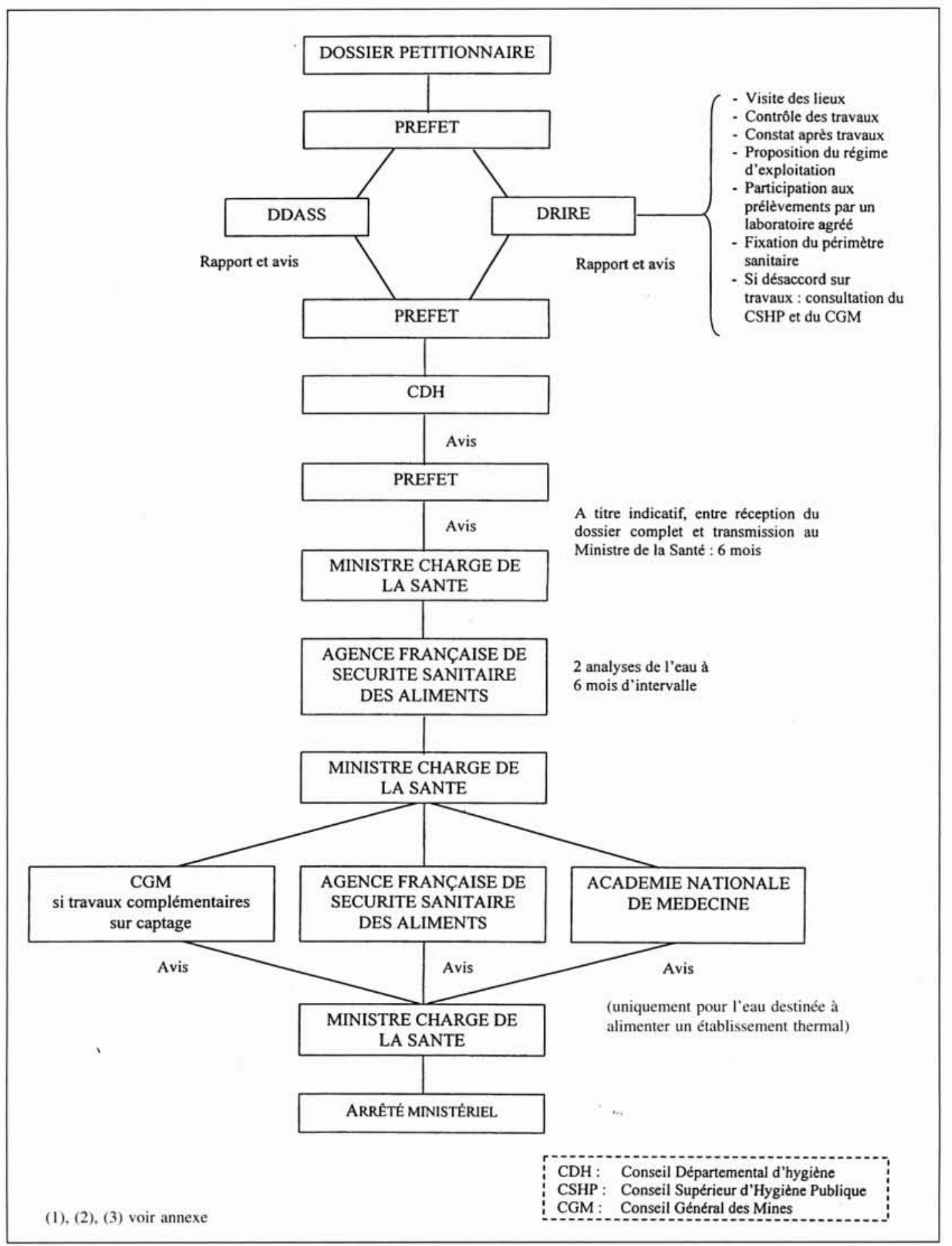

1. Procédure d'autorisation d'exploiter une source d'eau minérale.

Les premières autorisations délivrées en application de l'ordonnance de 1823, ont une validité illimitée. Cependant, à partir du 10 avril 1900, sur proposition de l'Académie de Médecine, le ministre limite la durée d'autorisation à 30 ans. Toutefois, aucun texte ne précise quelles formalités doit remplir le propriétaire d'une source après l'échéance de validité trentenaire. A défaut de consignes claires, le renouvellement est instruit comme une nouvelle demande d'autorisation.

A partir de 1957, les autorisations préalables à l'exploitation des eaux minérales deviennent plus nombreuses et les procédures plus compliquées. En effet, le décret 57-404 du 28 mars 1957, exige des exploitants de disposer de plusieurs autorisations ministérielles :
- exploitation de l'eau minérale à l'émergence, chaque captage étant considéré comme une source indépendante et, de ce fait, soumis à une procédure d'instruction complète ;

- transport par canalisation, en sachant que même un tuyau de quelques mètres alimentant une buvette doit être considéré comme une canalisation de transport ;

- mélange d'eaux minérales provenant de captages différents, ne pouvant être admises dans le mélange que des eaux provenant de sources déjà autorisées ayant des propriétés thérapeutiques et des caractéristiques physico-chimiques analogues et ayant une même origine géologique ;

- traitements éventuels (dégazéification, regazéification, décantation, déferrisation). 
En outre, chacune de ces autorisations doit faire l'objet d'une procédure de révision dès qu'il y a modification des conditions d'exploitation (modifications au niveau du captage, du débit, de la qualité de l'eau, de la canalisation de transport, du procédé de traitement, etc.).

Pour les eaux minérales conditionnées, le décret 64-1255 du 11 décembre 1964 impose également des autorisations ministérielles concernant l'embouteillage et l'utilisation des matériaux pour la fabrication des récipients, s'ils ne sont pas en verre.

En application de la loi sur l'eau 92-3 du 3 janvier 1992 , les eaux minérales sont soumises également à une procédure qui, suivant le lieu et la quantité d'eau prélevée dans le gisement souterrain, relève d'une déclaration ou d'une autorisation préfectorale après enquête publique.

La délivrance d'une autorisation requise par le décret de 1957 est précédée d'une instruction dont la procédure est longue et complexe. A titre d'exemple, sur la figure 1 est présenté le schéma du déroulement d'une procédure d'autorisation d'exploiter à l'émergence, dont la durée est, dans le meilleur des cas, de l'ordre de 15 à 20 mois.

Cette accumulation des décisions administratives auxquelles sont soumises les eaux minérales, a conduit les pouvoirs publics à envisager une révision de la réglementation qui devrait voir le jour en l'an 2000.

\section{III — PROTECTION DE LA RESSOURCE}

La sensibilisation au problème de la "conservation des eaux minérales" se manifeste déjà au début du XVIIIe siècle, lorsque, suite à des travaux souterrains à proximité de quelques sources, leur débit s'est trouvé perturbé. Cela a motivé la mise en place d'une législation sur les périmètres de protection, qui a vu le jour au milieu du XIXe siècle.

La préoccupation en termes de qualité sanitaire ("hygiénique") de l'eau minérale est apparue bien plus tard, vers la fin du XIXe siècle, après les découvertes scientifiques dans le domaine de la microbiologie. Il s'est avéré que certaines sources captées dans des conditions précaires et situées dans un environnement défavorable, étaient particulièrement vulnérables à la contamination bactériologique.

Les deux problèmes étant apparus historiquement à des époques différentes, ils ont donné lieu à deux réglementations distinctes complémentaires.

\section{- 3.1 Périmètre de protection}

Les premières zones de protection autour des sources minérales ont été créées par des décisions isolées. La plus ancienne connue est celle des bains de Balaruc (Hérault) dont les propriétaires ont vu leurs sources se tarir suite à des travaux réalisés par un voisin qui "par envie" a entrepris de "faire perdre les sources sous prétexte de faire un puits dans son propre fonds". Le procès engagé par les propriétaires des sources s'est soldé par un arrêt du Conseil d'État du 14 décembre 1715, qui, dans un espace délimité, défend "de faire aucun puits, fossés, creux ni excavations ou fondations de maisons, ni autres ouvrages qui puissent préjudicier aux eaux des bains."

Un autre arrêt du Conseil d’État du 6 mai 1732, concerne les bains de Barèges (Hautes Pyrénées) ; il fait "défenses à tous particuliers et propriétaires des terrains de construire, à l'avenir, aucune sorte d'édifice et bâtiments dans ledit hameau", sans permission préalable.
Au début du XIXe siècle, avec l'arrivée des techniques de forage, les tentatives de détournement des eaux alimentant les sources se multiplient. La "guerre des sources" fait son apparition dans plusieurs localités, les plus connues étant celles de Vichy et Saint Galmier. La nécessité d'instaurer des périmètres de protection autour des sources exploitées, devient urgente. Le premier projet de loi présenté en 1837 provoque des débats passionnés : les servitudes imposées aux tiers qui ont des terrains à l'intérieur du périmètre de protection, sont jugées inacceptables. Entre 1837 et 1847 , aucun des trois projets de loi, n'a réuni une majorité de la Chambre des députés. La révolution de 1848 ayant éclaté sur ces entrefaites et, par ailleurs, les sources de Vichy (appartenant à l'État) se trouvant sérieusement menacées par des sondages destinés manifestement à les détourner, le Gouvernement Provisoire prend la décision, par décret du 8 mars 1848, de fixer arbitrairement, autour de chaque source régulièrement autorisée, un périmètre de $1000 \mathrm{~m}$ de rayon, dans lequel "aucun sondage, aucun travail souterrain ne pourront être pratiqués sans l'autorisation préalable du préfet du département."

Toutefois, ce décret étant fortement contesté, un quatrième projet est élaboré et, après plusieurs amendements, la loi est adoptée et promulguée le 14 juillet 1856.

Aux termes de celle-ci, les sources d'eau minérale peuvent être déclarées d'intérêt public (les mots "utilité publique" étaient employés dans les projets) par décret en Conseil d'État. Dans les mêmes conditions, un périmètre de protection peut être assigné aux sources déclarées d'intérêt public. Mais à la différence des dispositions du décret de 1848 qui instituait autoritairement un périmètre de protection autour de chaque source régulièrement autorisée, la loi laisse l'initiative au propriétaire d'une source, de faire la demande et de présenter des arguments justifiant 1' "intérêt public" de sa source et motivant l'étendue du périmètre de protection.

A l'intérieur du périmètre, les sondages et travaux souterrains sont soumis à autorisation préalable du ministre. Le propriétaire de la source a la possibilité, après autorisation du ministre, d'occuper les terrains d'autrui pour réaliser "tous les travaux de captage et d'amélioration nécessaires pour la conservation, la conduite et la distribution" de cette source ; cependant en aucun cas l'expropriation ne peut avoir lieu.

La loi prévoit la publication de plusieurs décrets concernant les conditions de fixation des périmètres de protection, l'organisation de la surveillance des sources et des établissements et les conditions générales d'ordre et de police dans ces établissements. Ces décrets sont parus en 1856, 1860, 1930 et 1957.

Après quelques modifications mineures, la loi de 1856 a été codifiée (art. L735 à L751 du code de la santé publique) par décret 53-1001 du 5 octobre 1953, puis abrogée par la loi 58-346 du 3 avril 1958.

Initialement, tant le décret de 1848 que la loi de 1856, avaient pour objectif de protéger les sources, principalement sur le plan quantitatif : seuls pouvaient être réglementés les travaux susceptibles de détourner les eaux minérales. L'aspect qualitatif n'a été réellement pris en compte qu'à partir de 1992, avec la loi sur l'eau 92-3 du 3 janvier 1992, qui a modifié les articles L736 et L737 du code de la santé publique en soumettant également à autorisation préalable les "activités, dépôts ou installations de nature à nuire directement ou indirectement à la qualité des eaux...". 


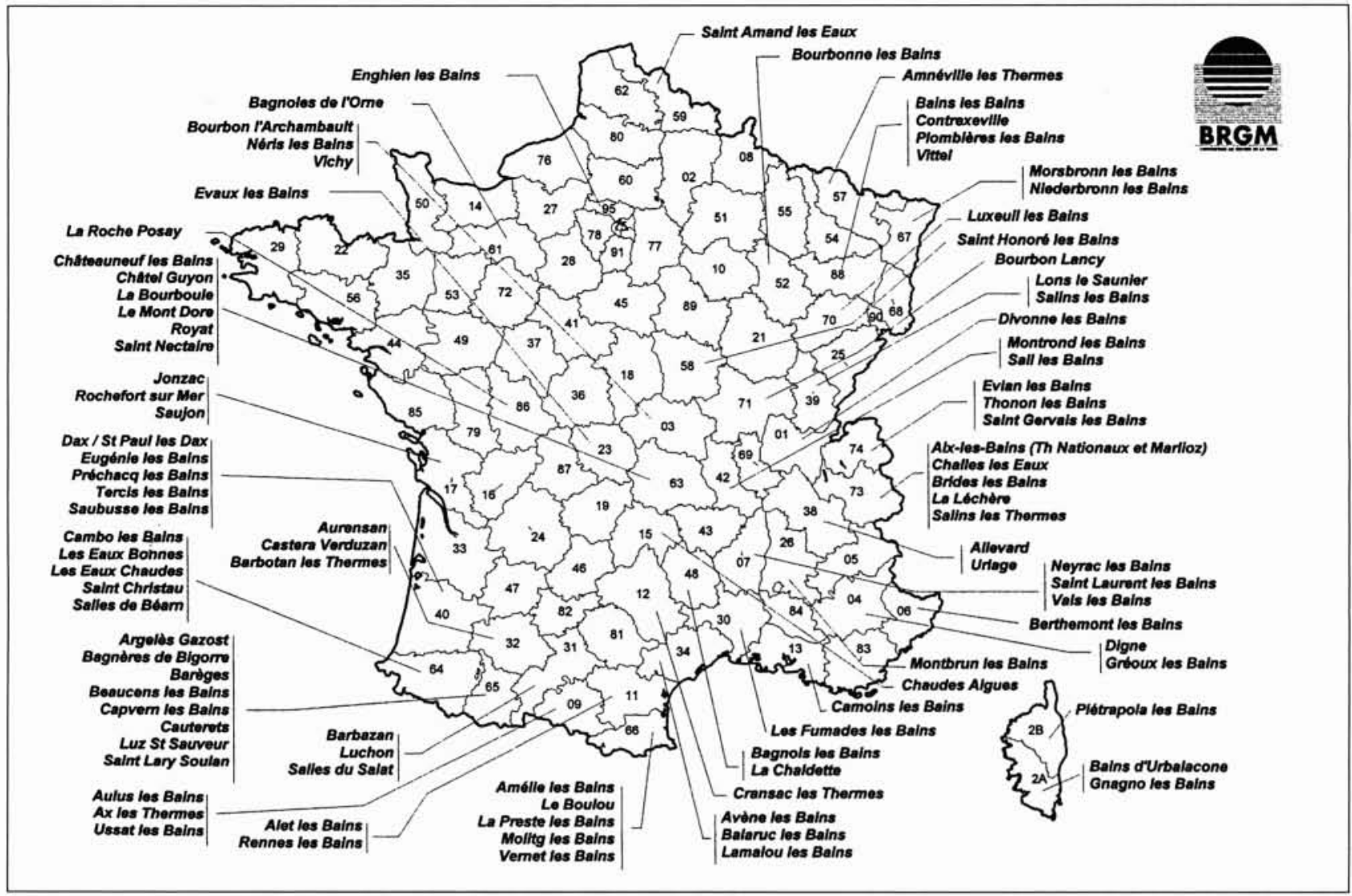

2. Répartition départementale des établissements thermaux en France métropolitaine.

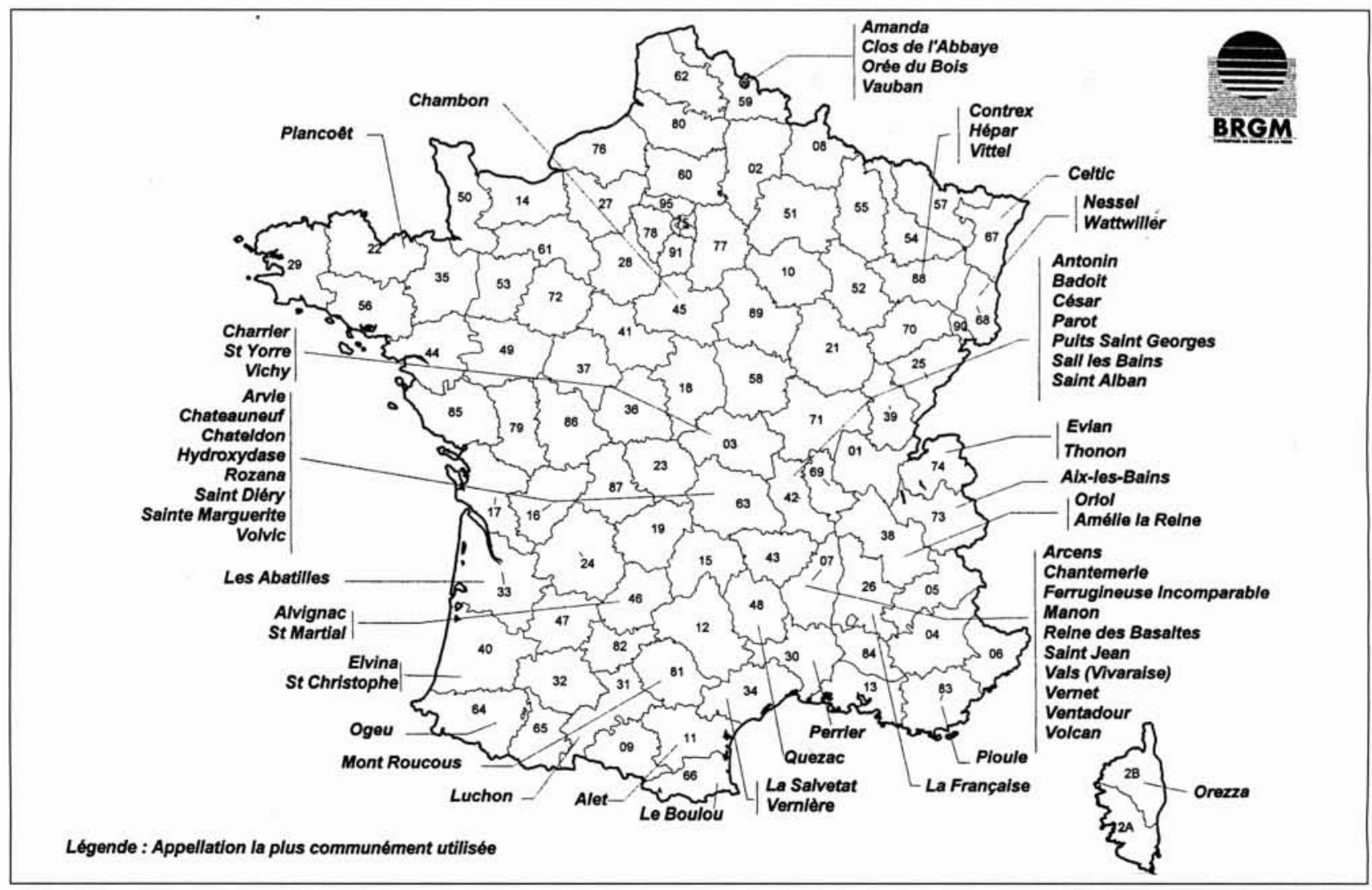

3. Répartition départementale des sites de production d'eau minérale naturelle embouteillée en France métropolitaine. 


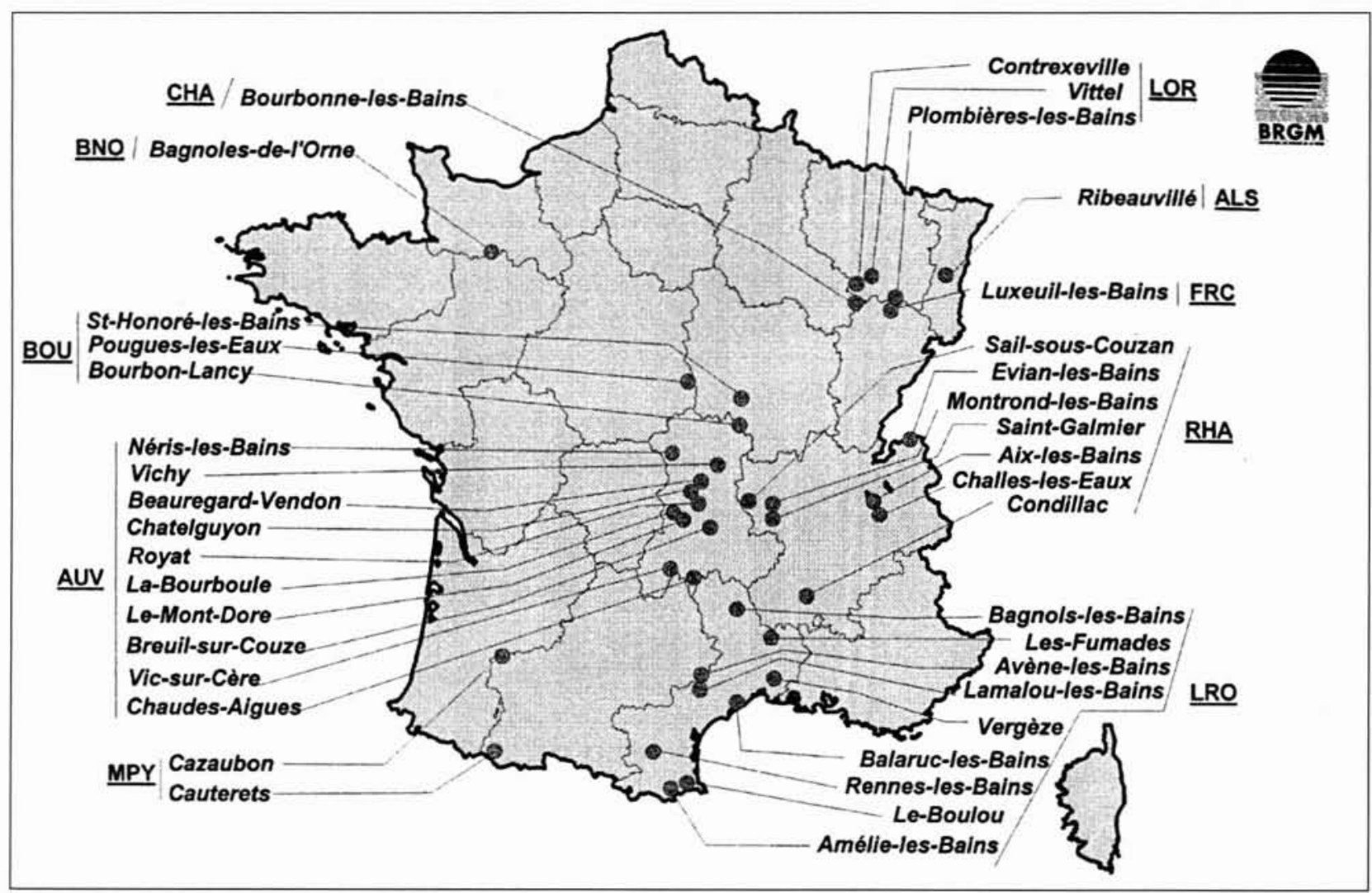

4. Répartition régionale des périmètres de protection des sources d'eau minérale en France métropolitaine.

Actuellement, sur le territoire national on dénombre 400 sources d'eau minérale naturelle exploitées sur 140 sites (fig 2 et 3 ) dont certains sont communs au thermalisme et à l'industrie d'embouteillage. De l'ordre de 160 sources bénéficient d'une déclaration d'intérêt public et 52 périmètres de protection ont été institués intéressant 48 sites (fig 4), certains d'entre eux étant concernés par 2 ou 3 périmètres (Vichy, Lamalou-les-Bains, Les Fumades). En fait, le nombre total des sources protégées, car situées à l'intérieur des périmètres, s'élève à 290.

En principe, le périmètre de protection ayant pour but de protéger la ressource en eau minérale, son étendue devrait englober l'ensemble du gisement. Dans la pratique courante, compte tenu de l'importance des servitudes et des contraintes pesant sur les terrains concernés, un compromis est systématiquement recherché entre ce qui est souhaitable et ce qui est strictement nécessaire. En général, les périmètres sont des polygones mais il en existe plusieurs de forme circulaire. Les superficies sont très variables, elles s'échelonnent entre 1 ha et 15600 ha, ce dernier étant attribué au bassin de Vichy.

\subsection{Périmètres sanitaires d'émergence}

La première circulaire qui fait état des risques de pollution des eaux minérales au point de captage, date du 9 août 1894 . Le ministre attire l'attention des préfets sur "le danger que fait courir aux consommateurs l'impureté possible de certaines eaux minérales" et exige que les griffons soient bien isolés et les vasques des sources recouvertes. Il ne s'agit dans cette circulaire que du captage et de la manière de dis- tribuer l'eau. L'aspect environnemental dans le voisinage du captage est pris en considération seulement en 1937 dans la circulaire du 18 juin dans laquelle le ministre recommande qu'à l'occasion de toute demande d'autorisation d'exploiter une nouvelle source d'eau minérale, le service des mines ainsi que le conseil départemental d'hygiène, déterminent une zone autour du captage, qui constituerait le "périmètre sanitaire d'émergence strictement indispensable pour éviter toute pollution proche". Le pétitionnaire doit être propriétaire des terrains concernés par ce périmètre ou acquérir sur eux des droits de servitude.

L'obligation de détenir des droits de propriété sur les terrains nécessaires à l'établissement du périmètre sanitaire d'émergence est confirmée dans le décret du 28 mars 1957.

Ce dispositif, mis en œuvre dès 1937, concerne toutes les sources d'eau minérale autorisées depuis cette date. Pour les sources autorisées antérieurement, cette mesure s'avère parfois difficilement applicable. En effet, par principe, l'usage de l'eau minérale devait se faire le plus près possible du point d'émergence, ce qui a motivé l'édification des premiers établissements à proximité des sources. Ainsi, de nombreux captages se sont trouvés entourés de bâtiments d'exploitation eux-mêmes enclavés dans des zones urbanisées, de sorte qu'il est souvent impossible d'établir un périmètre sanitaire d'émergence au sens strict. C'est pourquoi, sous peine d'être obligé d'abandonner une source et faire cesser l'activité thermale, il est parfois nécessaire de recourir à un compromis consistant à réduire le périmètre à l'ouvrage de captage ou à l'abri dans lequel il est situé. 


\section{IV — ÉVOLUTION ATTENDUE AU COURS DES PROCHAINES ANNÉES}

L'année 2000 devrait voir la parution d'une nouvelle réglementation concernant la gestion de la ressource, les conditions d'exploitation et les contrôles. Le nouveau règlement doit se substituer, partiellement ou totalement, à quatre décrets actuellement en vigueur. Les principales modifications consistent à :

- réduire le nombre de procédures d'autorisation ;

- simplifier la procédure d'autorisation relevant de la compétence du ministre :

- renforcer le contrôle de la qualité de l'eau livrée au public ;

- intégrer les dispositions de la loi sur l'eau ;

- achever la transposition dans le droit français des dispositions de la directive européenne relative aux eaux minérales naturelles embouteillées.

La définition de l'eau minérale naturelle retenue dans la nouvelle réglementation est celle de la directive 80/777/CEE du 15 juillet 1980, adaptée aux eaux minérales utilisées dans le thermalisme.

D'après le nouveau règlement, la "source" pourra être constituée de plusieurs émergences, en quoi il y a similitude avec la notion de source des anciens qui admettaient que plusieurs griffons puissent faire partie d'une source.

L'exigence de la preuve des effets favorables à la santé sera maintenue, uniquement pour les eaux destinées à être utilisées dans un établissement thermal. En effet, dans son arrêt du 17 juillet 1997, la Cour de Justice des Communautés Européennes a décidé que la démonstration de ces propriétés n'est pas obligatoire pour les eaux minérales naturelles embouteillées (les dispositions de la directive “doivent être interprétées en ce sens qu'elles s'opposent à ce qu'un État membre exige qu'une eau ait des propriétés favorables à la santé pour être reconnue comme eau minérale naturelle").

Resteront soumis à la procédure d'autorisation, uniquement l'exploitation à l'émergence et l'embouteillage, ces autorisations étant délivrées respectivement par le ministre et le préfet. La première autorisation sera en quelque sorte une reconnaissance en tant qu'eau minérale naturelle au sens de la directive et vaudra également autorisation au titre de la loi sur l'eau.
Le transport par canalisation et le stockage de l'eau minérale, de même que sa distribution en buvette publique, seront soumis à une déclaration au préfet.

Comme par le passé, les eaux minérales naturelles pourront subir certains traitements. L'éventail des traitements admis, dont la liste exhaustive paraîtra dans un arrêté, sera certainement élargi pour tenir compte des recommandations de l'OMS, des normes mondiales (Codex Alimentarius) et des critères de qualité fixés par la directive européenne, visant à limiter la teneur de certains éléments naturellement présents dans l'eau, mais qualifiés d' "indésirables" comme par exemple le fluor, le manganèse ou l'arsenic.

Dans une deuxième phase seront modifiés les textes relatifs à la protection de la ressource. En effet, force est de constater que le nombre de sources d'eau minérale protégées par des périmètres de protection est encore insuffisant et ceux qui ont été institués méritent, pour la plupart, d'être révisés pour être mieux adaptés aux gisements à protéger. Pour inciter les exploitants à faire les démarches dans ce sens, la modernisation des procédures, établies en 1856 puis modifiées en 1930, s'avère nécessaire.

\section{BIBLIOGRAPHIE}

[1] Ordonnance du 18 juin 1823

[2] Loi du 14 juillet 1856

[3] Rapport présenté par M. LELUT, Député du Corps Législatif, à la séance du 14 mai 1856 de la Chambre des Députés

[4] Dictionnaire général des eaux minérales et d’hydrologie médicale. J.B. BAILLIERE et FILS. Libraires de l'Académie Impériale de Médecine [1860]

[5] La législation en vigueur sur les eaux minérales par J. SABADEL. RICARD Frères, imprimeurs de la préfecture à Montpellier - [1865]

[6] Historique de la législation des eaux minérales naturelles et artificielles par Georges MAILLARD. Typographie CHAMEROT et RENOUARD $-[1891]$

[7] Législation et réglementation des eaux minérales et des stations thermales et climatiques - 1781 à 1924. Chambre Syndicale du Commerce et de l'Industrie des eaux minérales et établissements thermaux - [1924]

[8] Décrets des 8 septembre 1856, 28 janvier 1860, 30 avril 1930, 28 mars 1957. 11 décembre 1964 et 6 juin 1989

[9] Annales des Mines - Réalités Industrielles - Mai 1998

\section{ANNEXE}

(1) La substitution de l'Agence française de sécurité sanitaire des aliments au laboratoire national de la santé publique, introduite par le premier alinéa de l'article 7 du décret n 99-242 du 26 mars 1999, est entré en vigueur à la date d'uverture de la gestion de l'établissement (cf article 33 du décret $n^{\circ}$ 99-242), c'est-à-dire le ler avril 1999. En effet, la date d'ouverture de la gestion de l'établissement correspond, selon les dispositions du 2ème alinéa de T'article 29 de la loi $\mathrm{n}^{\circ}$ 98-535 du ler juillet 1998 relative au renforcement de la veille sanitaire et du contrôle de la sécurité sanitaire des produits destinés à l'homme, à la date de publication du décret portant nomination du directeur général de l'Agence française de sécurité sanitaire des aliments. Cette nomination a été prononcée par décret du 31 mars 1999, publié au Journal Officiel du ler avril 1999.

(2) La consultation de l'Agence française de sécurité sanitaire des aliments prévue par l'article 7 du décret n $99-242$ du 26 mars 1999 , entre en vigueur à compter de la date de publication du premier arrêté portant nomination des membres des comités d'experts spécialisés prévus à l'article R794-23 du code de la santé publique (cf article 33 du décret $n^{\circ}$ 99-242).

Cet arrêté daté du 30 août 2000 a été publié au Journal Officiel du 9 septembre 2000.

(3) Le 2ème alinéa de l'article 7 du décret n $99-242$ du 26 mars 1999 modifiant l'article 8 du décret n $57-404$ du 26 mars 1957 stipule que l'avis de l'Académie (nationale) de médecine n'est sollicité que si l'eau est destinée à alimenter un établissement thermal. Cependant, cette disposition n'entre en vigueur qu'à compter de la date de publication du premier arrêté portant nomination des membres des comités d'experts spécialisés prévus à l'article R794-23 du code de la santé publique (cf article 33 du décret n 99-242).

Cet arrêté daté du 30 août 2000 a été publié au Journal Officiel du 9 septembre 2000. 